\title{
Microscopic description of information transfer from a qudit to reservoir
}

\author{
Peter Štelmachovič ${ }^{1}$, Mário Ziman ${ }^{1}$, and Vladimír Bužek ${ }^{1,2, *}$ \\ ${ }^{1}$ Research Center for Quantum Information, Slovak Academy of Sciences, Dúbravská cesta 9, \\ 84228 Bratislava, Slovakia \\ ${ }^{2}$ Faculty of Informatics, Masaryk University, Botanická 68a, 60200 Brno, Czech Republic
}

Received 13 May 2002, accepted 21 May 2002

Published online 30 April 2003

\begin{abstract}
Recently Ziman et al. [3] have introduced a concept of a universal quantum homogenizer which is a quantum machine that takes as an input a system qubit initially in an arbitrary state $\rho$ and a set of $N$ reservoir qubits initially prepared in the same state $\xi$. The homogenizer realizes, in the limit sense, the transformation such that at the output each qubit is in an arbitrarily small neighbourhood of the state $\xi$ irrespective of the initial states of the system and the reservoir qubits. In this paper we generalize the concept of quantum homogenization for qudits, that is, for $d$-dimensional quantum systems. We prove that the partial swap operation induces a contractive map with the fixed point which is the original state of the reservoir. Finally we propose an optical realization of the quantum homogenization.
\end{abstract}

\section{Introduction}

One of the most fundamental problems in quantum theory is dynamics of open systems [1]. In general, one can assume an interaction between the open system denoted as $S$ with the environment $R$. This environment is a quantum system with the Hilbert space of an arbitrary dimension. The whole $S+R$ system evolves unitarily and the question of irreversibility of dynamics of open systems is then a great issue. How from a unitary evolution a irreversible dynamics can emerge? For instance, when a system interacts with a reservoir which is in thermal equilibrium then after some time the system is thermalized - it relaxes towards the thermal equilibrium. This implies that the information about the original state of the system is (irreversibly) "lost" and its new state is determined exclusively by the parameters (temperature) of the reservoir. If the reservoir is composed of a large number, $N$, of physical objects of the same physical origin as the system itself, then the thermalization process can be understood as homogenization: out of $N$ objects (the reservoir) prepared in the same thermal state and a single system in an arbitrary state, we obtain $N+1$ objects in the same thermal state. This intuitive picture is based on certain assumptions about the interaction between the system and the reservoir, about the physical nature of the reservoir itself and the concept of the thermal equilibrium. Such model is very important for understanding of many processes in quantum physics as well as the fundamental problem of the irreversibility [1,2]. For this reason it is important to analyze rigorously the process of information transfer in this simple model which has been first analyzed in a recent paper [3] for qubits. In this paper we present a rigorous analysis of the above picture within the framework of quantum information theory for $d$-dimensional quantum systems - qudits.

Specifically, we will consider a system, $S$, represented by a single qudit initially prepared in the unknown state $\varrho_{S}^{(0)}$, and a reservoir, $R$, composed of $N$ qudits all prepared in the state $\xi$, which is arbitrary but same for all qudits. We will enumerate the qudits of the reservoir and denote the state of the $k$-th qudit as $\xi_{k}$ [4].

\footnotetext{
* Corresponding author E-mail: buzek@savba.sk
} 


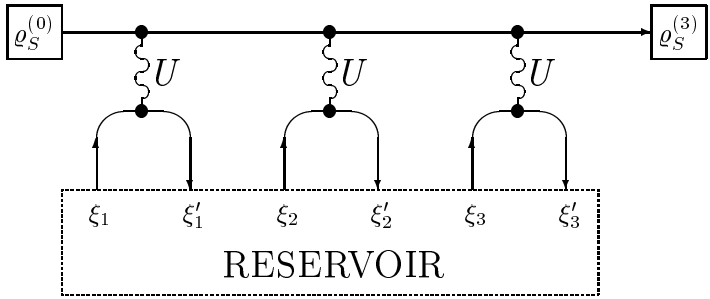

Fig. 1 Simple collision-like model of homogenization with just three reservoir qudits involved.

From the definition of the reservoir it follows that initially $\xi_{k}=\xi$ for all $k$, so the state of the reservoir is described by the density matrix $\xi^{\otimes N}$.

Let $U$ be a unitary operator representing the interaction between a system qudit and one of the reservoir qudits. In addition, let us assume that at each time step the system qudit interacts with just a single qudit from the reservoir (see Fig. 1). Moreover, the system qudit can interact with each of the reservoir qudits at most once. After the interaction with the 1-st reservoir qudit the system is changed according to the following rule (which is a completely-positive map)

$$
\varrho_{S}^{(1)}=\operatorname{Tr}_{1}\left[U \varrho_{S}^{(0)} \otimes \xi_{1} U^{\dagger}\right]
$$

Let us repeat the interaction $N$ times, that is, via a sequence of interactions the system qudit interacts with $N$ reservoir qudits all prepared in the state $\xi$. The final state of the system is then described by the density operator

$$
\varrho_{S}^{(N)}=\operatorname{Tr}_{R}\left[U_{N} \ldots U_{1}\left(\varrho_{S}^{(0)} \otimes \xi^{\otimes N}\right) U_{1}^{\dagger} \ldots U_{N}^{\dagger}\right]
$$

where $U_{k}:=U \otimes\left(\otimes_{j \neq k} \mathbf{1}_{j}\right)$ describes the interaction between the $k$-th qudit of the reservoir and the system qudit. This model of homogenization is very similar to the collision model since the system becomes homogenized via a sequence of individual interactions with the reservoir qudits. The interactions are assumed to be localized in time (i.e., they act like elastic collisions) [5].

Our aim is to investigate possible maps induced by the transformation (2) and describe the process of homogenization. Homogenization means that due to the interaction $U$ the states of the qudits in reservoir change only little while after $N$ interactions the system's state become close to the initial state of the reservoir qudits. Formally,

$$
\begin{aligned}
& \forall k, 1 \leq k \leq N \ldots D\left(\xi_{k}^{\prime}, \xi\right) \leq \delta ; \\
& \forall N \geq N_{\delta} \ldots D\left(\varrho_{S}^{(N)}, \xi\right) \leq \delta,
\end{aligned}
$$

where $D(\ldots, \ldots)$ denotes some distance (e.g., a trace norm) between the states, $\delta>0$ is a small parameter which is chosen a priori to determine the degree of the homogeneity and $\xi_{k}^{\prime}:=\operatorname{Tr}_{S}\left[U \varrho_{S}^{(k-1)} \otimes \xi U^{\dagger}\right]$ is the state of the $k$-th reservoir qudit after the interaction with the system qudit.

We note that homogenization is closely related to thermalization [6]. There are however two main differences: in thermalization, (i) the state $\xi$ of the reservoir qudits is not completely unknown, but is a thermal state, that is, a state diagonal in a given basis (interpreted as the basis of the eigenstates of a onequdit Hamiltonian); and (ii) the number of qudits in the reservoir is considered to be infinite for any practical purpose.

Our paper is organized as follows: in Sect. 2 we show that quantum homogenization can be realized with the help of a partial swap operation. In Sect. 3 we show that the partial swap for qudits generates a contractive map on the system qudit with the fixed point being the initial state of the reservoir. This ensures the required convergence of the homogenization process [see Eqs. (4) and (3)]. In Sect. 4 we estimate the fidelity of the approximate homogenization map as a function of the number $N$ of reservoir qudits and 
the parameter $\delta$ (the precision of the homogenization), while in the final Sect. 5 of the paper we address a feasible optical realization of the homogenization map for continuous variables.

\section{Partial-swap operation}

Let us start with the definition of the so-called swap operation $S$ acting on the Hilbert space of two qudits which is given by the relation [7]

$$
S|\psi\rangle \otimes|\phi\rangle=|\phi\rangle \otimes|\psi\rangle .
$$

With this transformation

$$
S \varrho^{(0)} \otimes \xi S^{\dagger}=\xi \otimes \varrho^{(0)},
$$

after just a single interaction, the state of the system $S$ is equal to the state $\xi$ of the reservoir qudit; and the interacting qudit from the reservoir is left in the initial state of system. This means the condition (4) is fulfilled, while the condition (3) is not - since recall that we want it to hold for all $\varrho^{(0)}$.

In order to fulfill both conditions (4) and (3) we have to find some unitary transformation which is "close" to the identity on the reservoir qudit, while it performs a partial swap operation, so that the system qudit at the output is closer to the reservoir state $\xi$ than before the interaction. The swap operator is Hermitian and therefore we can define the unitary partial swap operation

$$
P(\eta)=\cos \eta \mathbf{1}+i \sin \eta S
$$

that serves our purposes. In what follows we denote $\sin \eta=s$ and $\cos \eta=c$.

In the process of homogenization, the system qudit interacts sequentially with one of the $N$ qudits of the reservoir through the transformation $P(\eta)$. The states of the system qudit and of the reservoir qudit are obtained by partial traces. Specifically, after the first interaction the system qudit is in the state described by the density operator

$$
\varrho_{S}^{(1)}=c^{2} \varrho_{S}^{(0)}+s^{2} \xi+i c s\left[\xi, \varrho_{S}^{(0)}\right],
$$

while the first reservoir qudit is now in the state

$$
\xi_{1}^{\prime}=s^{2} \varrho_{S}^{(0)}+c^{2} \xi+i c s\left[\varrho_{S}^{(0)}, \xi\right] .
$$

We can recursively apply the partial-swap transformation and after the interaction with the $n$-th reservoir qudit, we have

$$
\varrho_{S}^{(n)}=c^{2} \varrho_{S}^{(n-1)}+s^{2} \xi+i c s\left[\xi, \varrho_{S}^{(n-1)}\right],
$$

as the expression for the density operator of the system qudit, while the $n$-th reservoir qudit is in the state

$$
\xi_{n}^{\prime}=s^{2} \varrho_{S}^{(n-1)}+c^{2} \xi+i c s\left[\varrho_{S}^{(n-1)}, \xi\right] .
$$

\section{Homogenization is a contractive map}

In this section we show that $\varrho_{S}^{(N)}$ monotonically converges to $\xi$ for all parameters $\eta \neq 0$. This means, in particular, that the condition (4) does not put any constraint on $\eta$. To show this convergence, we use the Banach theorem [8] that concerns the fixed point of a contractive transformation. Let $\mathcal{S}$ be a space with a distance function $D(\ldots, \ldots)$. The transformation $\mathcal{T}$ is called contractive if it fulfills the inequality $D(\mathcal{T}[\varrho], \mathcal{T}[\xi]) \leq k D(\varrho, \xi)$ with $0 \leq k<1$ for all $\varrho, \xi \in \mathcal{S}$. A fixed point of the transformation $\mathcal{T}$ is an element of $\mathcal{S}$ for which $\mathcal{T}[\xi]=\xi$. The Banach theorem states that a contractive map has a unique fixed point [9], and that the iteration of the map converges to it, i.e. $\mathcal{T}^{N}[\varrho] \rightarrow \xi$ for each $\varrho \in \mathcal{S}$. We note that contractive transformations within the context of quantum information processing have been recently discussed also in Ref. [10]. 


\subsection{Definition of the distance}

A natural way how to define a distance in a Hilbert space is to use the norm induced with the scalar product of the Hilbert space. Let us consider a separable not necessary finite dimensional Hilbert space $\mathcal{H}_{A}$. We say that an operator $\mathbf{A}: \mathcal{H}_{A} \rightarrow \mathcal{H}_{A}$ is Hilbert-Schmidt operator if it is bounded and there exists orthonormal basis $\left\{\left|\varphi_{i}\right\rangle\right\}$ of the Hilbert space $\mathcal{H}_{A}$ such that

$$
\sum_{i=1}^{\infty} \| \mathbf{A}\left|\varphi_{i}\right\rangle \|^{2}<\infty .
$$

The set of all Hilbert-Schmidt operators $\mathbf{A}: \mathcal{H}_{A} \rightarrow \mathcal{H}_{A}$ is also a Hilbert space $\mathcal{B}$ with scalar product defined as

$$
(\mathbf{A}, \mathbf{B})_{\mathcal{B}}=\operatorname{Tr}\left(\mathbf{A}^{\dagger} \mathbf{B}\right)
$$

where $\mathbf{A}$ and $\mathbf{B}$ are two elements of the Hilbert space $\mathcal{B}$. The norm of an element $\mathbf{A}$ of the Hilbert space $\mathcal{B}$ induced with the scalar product $(., .)_{\mathcal{B}}$ is given by

$$
\|A\|_{\mathcal{B}}=\sqrt{(\mathbf{A}, \mathbf{A})_{\mathcal{B}}} .
$$

Let us note that the norm in Eq. (14) may be infinite even though the operator $\mathbf{A}$ is a bounded operator $\mathbf{A}: \mathcal{H}_{A} \rightarrow \mathcal{H}_{A}$. For example, if the element $\mathbf{A}$ is a unitary operator acting in an infinite dimensional Hilbert space $\mathcal{H}_{A}$ the norm in Eq. (12) of $\mathbf{A}$ is infinite (and consequently any unitary operator does not belong to the Hilbert space $\mathcal{B}$ ). One should not forget that the norm of $\mathbf{A}$ considered as an element of the Hilbert space $\mathcal{B}$ may not equal to the norm of the same element $\mathbf{A}$ considered as a bounded operator $\mathbf{A}: \mathcal{H}_{A} \rightarrow \mathcal{H}_{A}$. It can be shown (see Ref. [11]) that the convex set of all density operators $\mathcal{S}$ is a subset of $\mathcal{B}$. Now the distance between two elements $\rho, \sigma$ of a given Hilbert space $\mathcal{B}$ can be defined with the help of the norm presented in Eq. (14) as follows

$$
D(\rho, \sigma) \equiv\|\rho-\sigma\|_{\mathcal{B}} .
$$

Using the expression (14) for the norm induced with a scalar product one may derive a more convenient form for the distance now expressed via the trace operation:

$$
D^{2}(\rho, \sigma)=(\rho-\sigma, \rho-\sigma)_{\mathcal{B}}=\operatorname{Tr}\left(\rho^{\dagger} \rho-\rho^{\dagger} \sigma-\sigma^{\dagger} \rho+\sigma^{\dagger} \sigma\right) .
$$

\subsection{Stability of the reservoir}

The first condition of the homogenization (3) requires all reservoir qudits remain in the $\delta$-neighbourhood of their initial state $\xi$. That is, states of the individual qudits of the reservoir are "stable" during the systemreservoir interactions, that is, these states are not changed much. As we apply sequentially the same unitary transformation $U$ in the case of all reservoir qudits it holds that if

$$
D\left(\xi_{1}^{\prime}, \xi\right) \leq \delta
$$

for all initial states $\rho$ of the system then

$$
D\left(\xi_{i}^{\prime}, \xi\right) \leq \delta \quad \forall i=1 \ldots N .
$$

Apparently more natural reasoning would be to exploit the convergence $\rho^{(n)} \rightarrow \xi$ which follows from the contractivity of the map $\mathcal{T}$ proved in the following section. If the state $\rho^{(n)}$ converges monotonously to the state $\xi$ then

$$
D\left(\xi_{1}^{\prime}, \xi\right) \geq D\left(\xi_{2}^{\prime}, \xi\right) \geq D\left(\xi_{3}^{\prime}, \xi\right) \ldots
$$


which is a direct consequence of Eq. (11). However, one does not need the convergence to prove it. It simply follows from the fact that the relation Eq. (16) must hold for all initial states of the system $\rho$ and that we sequentially apply the same unitary transformation $U$ [see Eqs. (10) and (11)]. The important point is that it is sufficient to estimate only the distance $D\left(\xi_{1}^{\prime}, \xi\right)$. Using the expression (9) for the state $\xi_{1}^{\prime}$ one has

$$
D\left(\xi_{1}^{\prime}, \xi\right)=\left\|s^{2} \rho-s^{2} \xi+i c s[\rho, \xi]\right\|_{\mathcal{B}}
$$

The last result can be further simplified. Recall that for any two elements $\mathbf{A}$ and $\mathbf{B}$ from $\mathcal{B}$ equation

$$
\begin{aligned}
\|\mathbf{A}+i \mathbf{B}\|_{\mathcal{B}}^{2} & =(\mathbf{A}+i \mathbf{B}, \mathbf{A}+i \mathbf{B})_{\mathcal{B}}=(\mathbf{A}, \mathbf{A})_{\mathcal{B}}+(\mathbf{B}, \mathbf{B})_{\mathcal{B}}+i\left[(\mathbf{A}, \mathbf{B})_{\mathcal{B}}-(\mathbf{B}, \mathbf{A})_{\mathcal{B}}\right] \\
& =\|\mathbf{A}\|_{\mathcal{B}}^{2}+\|\mathbf{B}\|_{\mathcal{B}}^{2}+i\left[(\mathbf{A}, \mathbf{B})_{\mathcal{B}}-(\mathbf{B}, \mathbf{A})_{\mathcal{B}}\right]
\end{aligned}
$$

holds. If the scalar product $(\mathbf{A}, \mathbf{B})_{\mathcal{B}}$ is real, then the expression in the brackets equals to zero and one obtains

$$
\|\mathbf{A}+i \mathbf{B}\|_{\mathcal{B}}^{2}=\|\mathbf{A}\|_{\mathcal{B}}^{2}+\|\mathbf{B}\|_{\mathcal{B}}^{2}
$$

The scalar product $\left(s^{2} \rho-s^{2} \xi, c s[\rho, \xi]\right)_{\mathcal{B}}$ is apparently real and from Eqs. (20) and (21) it follows that

$$
D^{2}\left(\xi_{1}^{\prime}, \xi\right)=\left\|s^{2} \rho-s^{2} \xi\right\|_{\mathcal{B}}^{2}+\|i c s[\rho, \xi]\|_{\mathcal{B}}^{2} .
$$

Using the "law of parallelogram" (which holds for the norm induced with a scalar product)

$$
\|x+y\|^{2}+\|x-y\|^{2}=2\|x\|^{2}+\|y\|^{2}
$$

the first term in Eq. (22) can be rewritten as

$$
\left\|s^{2} \rho-s^{2} \xi\right\|_{\mathcal{B}}^{2}=s^{4}\left(2\|\rho\|_{\mathcal{B}}^{2}+2\|\xi\|_{\mathcal{B}}^{2}-\|\rho+\xi\|_{\mathcal{B}}^{2}\right) .
$$

The elements $\rho$ and $\xi$ are density matrices, i.e. positive operators with unite trace which gives certain bounds on the terms on the left side of Eq. (24):

$$
\begin{aligned}
\|\rho\|_{\mathcal{B}}^{2} & =\operatorname{Tr}(\rho \rho) \leq 1, \\
\|\xi\|_{\mathcal{B}}^{2} & \leq 1, \\
\|\rho+\xi\|_{\mathcal{B}}^{2} & \geq\|\rho\|_{\mathcal{B}}^{2}+\|\xi\|_{\mathcal{B}}^{2} .
\end{aligned}
$$

Inserting these results into Eq. (24) we can estimate the first term on the right side of the Eq. (22) as $\left\|s^{2} \rho-s^{2} \xi\right\|_{\mathcal{B}}^{2} \leq 2 s^{4}$. In the same way it can be shown that $\|i c s[\rho, \xi]\|_{\mathcal{B}}^{2} \leq 2 c^{2} s^{2}$ and we finally obtain

$$
D^{2}\left(\xi_{1}^{\prime}, \xi\right) \leq 2 s^{4}+2 c^{2} s^{2}=2 s^{2} .
$$

\subsection{Contractivity}

Consider two elements $\rho, \sigma \in \mathcal{S}$, i.e. two density matrices and denote $\rho-\sigma$ as $\rho-\sigma=\mathbf{A}$. The element $\mathbf{A}$ is hermitian (this follows from the fact that $\rho$ and $\sigma$ are density operators, that are bounded and self adjoint) and the distance between the two elements $\rho$ and $\sigma$ reads

$$
D^{2}(\rho, \sigma)=\|\mathbf{A}\|_{\mathcal{B}}^{2}=\operatorname{Tr}(\mathbf{A A})=\sum_{i, j}|\langle i|\mathbf{A}| j\rangle|^{2}
$$

Let us consider now two elements $\rho^{(1)}$ and $\sigma^{(1)}$, i.e. $\rho^{(1)}=\mathcal{T}[\rho]$ and $\sigma^{(1)}=\mathcal{T}[\sigma]$. Using the expression (15) for the distance $D(\ldots, \ldots)$ and the expression (8) for $\rho^{(1)}$ and $\sigma^{(1)}$ the distance between the two elements is given by

$$
\begin{aligned}
D\left(\rho^{(1)}, \sigma^{(1)}\right) & =D\left(c^{2} \rho^{(0)}+s^{2} \xi+i c s\left[\xi, \rho^{(0)}\right], c^{2} \sigma^{(0)}+s^{2} \xi+i c s\left[\xi, \sigma^{(0)}\right]\right) \\
& =\left\|c^{2} \rho^{(0)}-c^{2} \sigma^{(0)}+i c s\left[\xi, \rho^{(0)}-\sigma^{(0)}\right]\right\|_{\mathcal{B}}
\end{aligned}
$$


The last result can be again simplified using Eqs. (20) and (21). It is easy to check that

$$
\left(c^{2} \rho^{(0)}-c^{2} \sigma^{(0)}, i c s\left[\xi, \rho^{(0)}-\sigma^{(0)}\right]\right)_{\mathcal{B}}=i c^{3} s \times C
$$

so that the scalar product $\left(c^{2} \rho^{(0)}-c^{2} \sigma^{(0)}, \operatorname{cs}\left[\xi, \rho^{(0)}-\sigma^{(0)}\right]\right)_{\mathcal{B}}$ is real and consequently

$$
D^{2}\left(\rho^{(1)}, \sigma^{(1)}\right)=c^{4}\left\|\rho^{(0)}-\sigma^{(0)}\right\|_{\mathcal{B}}^{2}+c^{2} s^{2}\left\|\left[\xi, \rho^{(0)}-\sigma^{(0)}\right]\right\|_{\mathcal{B}}^{2} .
$$

Recall that $\rho^{(0)}$ is in fact $\rho$ (in same way $\sigma=\sigma^{(0)}$ ) so that $\rho^{(0)}-\sigma^{(0)}=\mathbf{A}$ and

$$
D^{2}\left(\rho^{(1)}, \sigma^{(1)}\right)=c^{4}\|\mathbf{A}\|_{\mathcal{B}}^{2}+c^{2} s^{2}\|[\xi, \mathbf{A}]\|_{\mathcal{B}}^{2}
$$

The second term in the last expression can be rewritten with the help of the scalar product [see Eq. (14)] or directly using a more convenient form in Eq. (16)

$$
\|[\xi, \mathbf{A}]\|_{\mathcal{B}}^{2}=2 \operatorname{Tr}\left(\xi^{2} \mathbf{A}^{2}\right)-2 \operatorname{Tr}(\xi \mathbf{A} \xi \mathbf{A}) .
$$

The operator $\xi$ is a density operator, which implies that the operator $\xi$ is compact. Every nonzero element of the spectrum of a compact operator is an eigenvalue. It means that every density operator $\xi$ can be written as $\xi=\sum_{i} \lambda_{i}|i\rangle\langle i|$ where $\lambda_{i}$ are non-zero eigenvalues of the operator $\xi$ and $|i\rangle$ are the corresponding eigenvectors. Let us perform the trace using a basis consisting of the eigenvectors $|i\rangle$ of the density matrix $\xi[12]$

$$
\begin{aligned}
\|[\xi, \mathbf{A}]\|_{\mathcal{B}}^{2} & =2 \sum_{i}\left\langle i\left|\xi^{2} \mathbf{A}^{2}\right| i\right\rangle-2 \sum_{i}\langle i|\xi \mathbf{A} \xi \mathbf{A}| i\rangle \\
& =2 \sum_{i, j} \lambda_{i}^{2}|\langle i|\mathbf{A}| j\rangle|^{2}-2 \sum_{i, j} \lambda_{i} \lambda_{j}|\langle i|\mathbf{A}| j\rangle|^{2}
\end{aligned}
$$

Since the operator $\mathbf{A}$ is hermitian it follows that $|\langle i|\mathbf{A}| j\rangle|^{2}=|\langle j|\mathbf{A}| i\rangle|^{2}$ and

$$
\begin{aligned}
\left.\|[\xi, \mathbf{A}]\|\right|_{\mathcal{B}} ^{2} & =\sum_{i, j} \lambda_{i}^{2}|\langle i|\mathbf{A}| j\rangle|^{2}+\sum_{i, j} \lambda_{j}^{2}|\langle i|\mathbf{A}| j\rangle|^{2}-2 \sum_{i, j} \lambda_{i} \lambda_{j}|\langle i|\mathbf{A}| j\rangle|^{2} \\
& =\sum_{i, j}\left(\lambda_{i}-\lambda_{j}\right)^{2}|\langle i|\mathbf{A}| j\rangle|^{2}
\end{aligned}
$$

Recall that $\lambda_{i}$ are non-zero eigenvalues of the density matrix $\xi$, i.e. positive and $\lambda_{i} \leq 1$ for all $i$. It follows that

$$
\left|\lambda_{i}-\lambda_{j}\right| \leq 1 \quad \forall i, j
$$

and

$$
\|[\xi, \mathbf{A}]\|_{\mathcal{B}}^{2} \leq\|\mathbf{A}\|_{\mathcal{B}}^{2}
$$

Inserting the last result into Eq. (30) together with the expression for the element $\mathbf{A}$ we obtain the following relation

$$
\begin{aligned}
D^{2}\left(\rho^{(1)}, \sigma^{(1)}\right) & \leq c^{4}\left\|\rho^{(0)}-\sigma^{(0)}\right\|_{\mathcal{B}}^{2}+c^{2} s^{2}\left\|\rho^{(0)}-\sigma^{(0)}\right\|_{\mathcal{B}}^{2} \\
& \leq c^{2} D^{2}\left(\rho^{(0)}, \sigma^{(0)}\right)
\end{aligned}
$$

which implies that the map $\mathcal{T}: \rho^{(0)} \rightarrow \rho^{(1)}$ is contractive iff $|c|<1$. 


\section{Approximate homogenization}

In what follows we will analyze homogenization not as the limit of the infinite number of interactions, but as an approximate process after a finite number of steps. Let us suppose that the parameter $\delta$ from Eqs. (4) and (3) is fixed. This parameter characterizes our approximation. We will use the partial-swap evolution for the description of the homogenization.

In the first step we give a condition on the parameter $\eta$ of the partial swap (7). For our map $\mathcal{T}$, we have that $D\left(\xi_{k}^{\prime}, \xi\right) \leq D\left(\xi_{1}^{\prime}, \xi\right)$. As we have discussed it earlier, we can adjust the parameter $\eta$ so that the condition $D\left(\xi_{1}^{\prime}, \xi\right) \leq \delta$ is fulfilled. Obviously, the distance $D\left(\xi_{1}^{\prime}, \xi\right)$ depends on the initial state of the system, $\varrho_{S}^{(0)}$, and on $\eta$. Therefore we have to determine the maximum value of $\eta$, for which the distance is less than or equal to $\delta$, independent (the universality condition) of the initial state of the system and reservoir. Using Eq. (26) we get the simple relation

$$
\sin \eta \leq \frac{\delta}{\sqrt{2}} .
$$

The second step is to determine the minimum number of interactions, $N$, that ensures for an arbitrary initial state of the system that the final state is in a sphere of radius $\delta$ around the reservoir state $\xi$. The worst case, i.e. when the number of necessary iterations is maximal, is intuitively the case when $D\left(\varrho_{S}^{(0)}, \xi\right)$ is maximal. In Sect. 3 we proved the convergence of the system state to $\xi$ for any $\eta \neq 0$. Therefore we are sure that such an $N$ exists. The value of $N$ can be obtained from Eq. (35)

$$
D\left(\rho_{S}^{(N)}, \xi\right)=|c|^{N} D\left(\rho_{S}^{(0)}, \xi\right)
$$

As was just discussed in the previous paragraph, the distance $D\left(\varrho_{S}^{(0)}, \xi\right)$ is maximal, when the two states are pure and mutually orthogonal and in this case $D\left(\varrho_{S}^{(0)}, \xi\right)=\sqrt{2}$ so that

$$
D\left(\rho_{S}^{(N)}, \xi\right)=\sqrt{2}|c|^{N}
$$

and the minimum number of reservoir qudits which are necessary to achieve the homogenization with a required fidelity is

$$
N \geq N_{\delta}=\frac{2 \ln (\delta / \sqrt{2})}{\ln \sqrt{\left(1-\delta^{2} / 2\right)}} .
$$

Both bounds on the parameters $\eta$ and $N$ are completely determined by the parameter $\delta$. After performing $N$ iterations, $N+1$ qudits are in states belonging to the $\delta$ neighborhood of the initial state of the reservoir, no matter what the states $\xi$ and $\varrho_{S}^{(0)}$ were. We see that if we fix the number of reservoir's qudits $N$, then the other two parameters are determined by the relations (38) and (36).

\section{Conclusions and discussion}

In this paper we have presented a simple model of an open system interacting with reservoir particles. We have shown that a partial swap operation induces on a system qudit a contractive map with the fixed point which is the initial state of reservoir qudits. This model can serve for a detailed analysis of an information transfer and the problem of reversibility from the point of view of quantum information theory. As shown in Ref. [3] the process of homogenization can be reversed and the original state of the system $\varrho_{S}^{(0)}$ and the reservoir $\xi$ can be recovered. only when the $N+1$ qubits of the output state interact, via the inverse of the original partial-swap operation, in the "correct" order. This classical information about the sequence of interaction is vital for reversibility of the quantum process under consideration. 
We conclude the paper by noting that our results are valid not only for qudits but also for continuous variables. That is, the model can be used for a description of an interaction of optical fields in an appropriate settings. In particular, the partial swap operation can be realized with the help of a beam splitter, so that the whole process of the homogenization can be represented as a transmition of a signal mode via a sequence of highly transmitive beam splitters. In an idler port of each of the beam splitter we consider an idler mode, playing the role of the reservoir particle. All these modes are initially in the same state $\xi$. It can be shown [13] that at the output of the sequence of the beam splitters the signal mode is in the state which (in the limit sense $N \rightarrow \infty$ ) is the state of the reservoir modes.

Acknowledgements We thank Mark Hillery, Valerio Scarani and Nicolas Gisin for discussions on quantum homogenization of qubits. This was work supported in part by the European Union projects EQUIP (IST-1999-11053), QUBITS (IST-1999-13021), and by the Slovak Academy of Sciences.

\section{References}

[1] E.B. Davies, Quantum Theory of Open Systems (Academic Press, London 1976).

[2] A. Peres, Quantum Theory: Concepts and Methods (Kluwer, Dordrecht 1993).

[3] M. Ziman, P. Štelmachovič, V. Bužek, M. Hillery, V. Scarani, and N. Gisin, Phys. Rev. A 65, 042105 (2002).

[4] We implicitly assume that the reservoir qudits are distinguishable. The validity of this assumption depends on the physical realization of the qudit. For instance, if the qudits are nuclear spins - or more generally, if each qudit is a degree of freedom of a given atom - the assumption is valid, since atoms are distinguishable from one another under normal conditions.

[5] R. Alicki and K. Lendi, Quantum Dynamical Semigroups and Applications, Lecture Notes in Physics (SpringerVerlag, Berlin 1987).

[6] V. Scarani, M. Ziman, P. Štelmachovič, N. Gisin, and V. Bužek, Phys. Rev. Lett. 88, 097905 (2002).

[7] See for example: M.A. Nielsen and I.L. Chuang, Quantum Computation and Quantum Information (Cambridge University Press, Cambridge 2000).

[8] M. Reed and B. Simon, Methods of Modern Mathematical Physics I: Functional Analysis (Academic Press, San Diego 1980).

[9] This is very easy to see: Suppose that $\xi$ and $\xi^{\prime}$ are two fixed points. Then they must satisfy $D\left(\xi, \xi^{\prime}\right) \leq k D\left(\xi, \xi^{\prime}\right)$ for a given $k \in\left[0,1\left[\right.\right.$. This is impossible unless $D\left(\xi, \xi^{\prime}\right)=0$.

[10] M. Raginski, Los Alamos arXiv quant-ph/0105141 (2001).

[11] J. Blank, M. Exner, and M. Havlíček, Linear Operators in Quantum Physics (UK Karolinium, Praha 1993).

[12] Note that the eigenvectors corresponding to the non-zero eigenvalues may not form a basis but only an orthonormal set of vectors. According to the Gram-Schmidt theorem every such set can be completed to form an orthonormal basis in the corresponding Hilbert space.

[13] D. Nagaj, P. Štelmachovič, M.S. Kim, and V. Bužek, Phys. Rev. A 66, 062307 (2002). 